\title{
Shared/Restricted Hybrid Access in Femtocells for Next Generation Wireless Networks
}

\author{
Muhammad Rehan Usman, Muhammad Arslan Usman, Sang Hoon Lee, Soo Young Shin* \\ WENS Lab, Department of IT Convergence, Kumoh National Institute of Technology, Yangho-Dong, Gumi, \\ Gyeongbuk-do, South Korea. \\ * Corresponding author. Tel.: +82-54-478-7473; email: wdragon@kumoh.ac.kr \\ Manuscript submitted October 7, 2014; accepted March 25, 2015. \\ doi: 10.17706/ijcce.2015.4.4.274-281
}

\begin{abstract}
In this paper, we have proposed two models, shared hybrid access (SHA) and restricted hybrid access (RHA), for the femtocell implementation in the next generation wireless cellular networks. The models proposed are based on the two dimensional Markov chain process. The Third Generation Partnership Project (3GPP) has described two kinds of users in the femtocell application; open users and the closed subscriber group (CSG) users referring to open access and closed access respectively. In this work, for the better explanation of the proposed models, we divide the open users and CSG users in two groups; the non subscriber group (NSG) and the subscriber group (SG) respectively. Both proposed models include the blocking probability analysis for SG and NSG user channels. For the validation of the proposed models, the simulation results of the derived blocking probabilities, for SG and NSG, are provided. We have also provided guidelines for the implementation of the proposed models, based on our simulation results, for improving the quality of service $(\mathrm{Q} O S)$, at the end of this paper.
\end{abstract}

Key words: Closed subscriber group (CSG), femtocell, hybrid access, mobile wireless networks.

\section{Introduction}

In next generation wireless cellular networks, the terms capacity, coverage and network congestion are of great importance. To stay in competition, network operators are working rigorously to improve these factors for enhancing the network's QoS for voice and data services. Data services are becoming more complex as they are characterized by a vast number of applications and protocols. More coverage and capacity in the existing cellular networks is needed in order to meet the requirements of exponentially increasing mobile traffic. Recently, the concept of femtocells has been presented as one of the solutions to overcome such issues. According to [1]-[3], the key technique to increase the spectral efficiency and network capacity is to overlay a macro cell base station with small cells, mainly femtocells.

Femtocell is small low power BS, known as femtocell access point (FAP) similar to a WiFi access point (AP) and the difference is that it provides feature of mobile phone access. It allows the network operators to extend indoor coverage especially at places where the access is unavailable or limited [4]. Till now the FAPs allow two kinds of access mechanisms; open access for the enterprise deployment and closed access for the residential users. In open access all the users of the core network are allowed access to the FAP whereas in closed access only a specific number of users, registered to the FAP, are allowed access to that FAP [5]. In [6]-[9] the performance of open and closed access has been studied in terms of resource allocation management in heterogeneous networks. 
The researchers are now working to provide access to both open and CSG users to the FAPs, known as hybrid access, for better capacity and coverage of the network. In hybrid access the open users are referred as non subscribers and CSG users as subscribers. As subscribes are the rightful owners of the FAP, so the main issue in the deployment of hybrid access is to keep the service quality of the subscribers unaffected by the non subscribers. Till now a number of studies have been done to improve the QoS of the femtocell users using hybrid access. In [10] the effect of the Mobile Stations (MS), that are within the range of the femtocell but are not communicating with it, is explored in terms of performance over a completely open or closed access. One of the strategies to improve QoS of the subscribers in hybrid access is to use orthogonal frequency division and multiple access (OFDMA) technique, as it reduces the cross-layer co-channel interference [5], [11]. In hybrid access, it is highly undesirable if the subscribers are denied access because of high congestion of non subscribers in the FAP area. To reduce the blocking probability of subscribers, in our work, we have extended the idea of shared and restricted access, presented in [5], in the form of two models; shared hybrid access (SHA) and restricted hybrid access (RHA).

The paper is organized in following manner. Section 2 consists of the proposed models with their respective blocking probability derivations using the Markov chain analysis, Section 3 consists of the simulation results with the QoS discussions for SHA and RHA and finally, the concluding remarks are provided in the Section 4.

\section{Proposed Models}

\subsection{Shared Hybrid Access (SHA) Model}

In this model all the channel resources are shared by both SG and NSG users. Whenever the channels are free they can be allocated to the NSG users. This model more likely follows first come first serve channel allocation strategy as both the SG and NSG users have same probability to be allocated channels.

In Fig. 1 we have total $s$ states where, $\lambda_{\mathrm{SG}}$ is the SG user arrival rate, $\lambda_{\mathrm{NSG}}$ is the NSG user arrival rate, $\mu_{\mathrm{SG}}$ is the SG user service rate and $\mu_{\mathrm{NSG}}$ is the NSG service rate. Service rate is the time for which a user remains connected to the system. From Fig. 1, $s$ states represent the total number of channels and all the channels from 0 to $s$ states are available to both SG and NSG users.

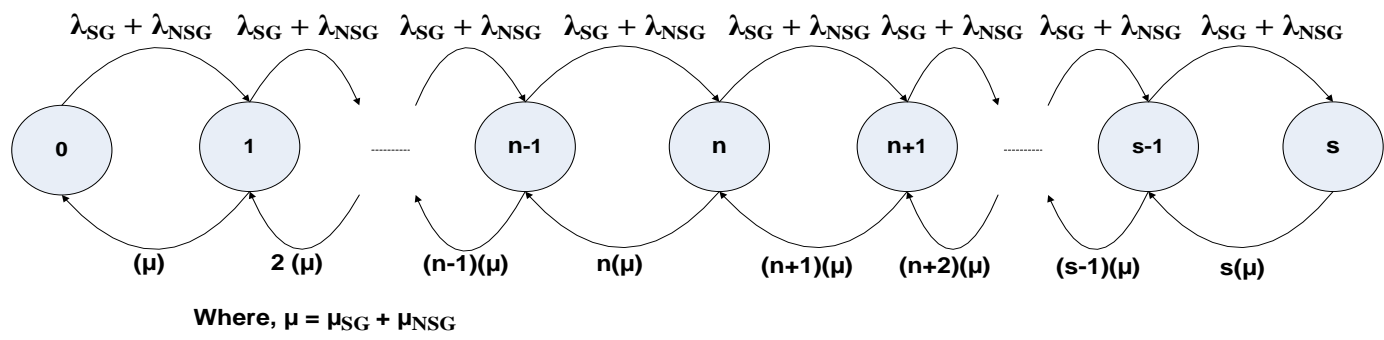

Fig. 1. Markov chain model for shared hybrid access (SHA).

Using the Fig. 1 and the basic equation work in [12] to find the total state probability, let us consider that $P_{j}$ is the statistical probability for total $j$ states, then for $0 \leq j<s$ the transition rate for $P_{j}$ to $P_{j+1}$ states is given by $\lambda_{\mathrm{SG}}+\lambda_{\mathrm{NSG}}$ and for $P_{j+1}$ to $P_{j}$ is given by $(j+1)\left(\mu_{\mathrm{SG}}+\mu_{\mathrm{NSG}}\right)$. We calculate the blocking probability for the $\mathrm{SG}$ users as follows:

$$
P_{j}=\left[\left(\frac{\lambda_{\mathrm{SG}}+\lambda_{\mathrm{NSG}}}{\mu_{\mathrm{SG}}+\mu_{\mathrm{NSG}}}\right)^{j} / j !\right] P_{o}
$$

To calculate the total probability of $j$ states we need to find out the probability of the zero state $P_{o}$. The 
zero state probability can be found by using the total state probability equation:

$$
\sum_{j=0}^{S} P_{j}=1
$$

By solving eq. (1) and eq. (2) we get:

$$
P_{0}=1 / \sum_{k=0}^{s} \frac{\left(\frac{\lambda_{\mathrm{SG}}+\lambda_{\mathrm{NSG}}}{\mu_{\mathrm{SG}}+\mu_{\mathrm{NSG}}}\right)^{a}}{a !}
$$

Putting the value of $P_{0}$ in eq. (1) we get:

$$
P_{j}=\frac{\left(\frac{\lambda_{\mathrm{SG}}+\lambda_{\mathrm{NSG}}}{\mu_{\mathrm{SG}}+\mu_{\mathrm{NSG}}}\right)^{j}}{j !} / \sum_{a=0}^{S} \frac{\left(\frac{\lambda_{\mathrm{SG}}+\lambda_{\mathrm{NSG}}}{\mu_{\mathrm{SG}}+\mu_{\mathrm{NSG}}}\right)^{a}}{a !}
$$

Then the total blocking probability equation for SG users will be given by replacing $s$ with $j$ in eq. (4):

$$
P_{b, \mathrm{SG}}=P_{S}=\frac{\left(\frac{\lambda_{\mathrm{SG}}+\lambda_{\mathrm{NSG}}}{\mu_{\mathrm{SG}}+\mu_{\mathrm{NGG}}}\right)^{s}}{s !} / \sum_{a=0}^{s} \frac{\left(\frac{\lambda_{\mathrm{SG}}+\lambda_{\mathrm{NSG}}}{\mu_{\mathrm{SG}}+\mu_{\mathrm{NSG}}}\right)^{a}}{a !}
$$

\subsection{Restricted Hybrid Access (RHA) Model}

In this model we have reserved channels resources for the SG users. These channels cannot be accessed by the NSG users. If the reserved channels are busy then the SG users can be connected to the remaining channels outside the reserved range.

Similarly, as in SHA model, $s$ states represent the total number of channels, where, $\lambda_{\mathrm{SG}}, \lambda_{\mathrm{NSG}}, \mu_{\mathrm{SG}}$ and $\mu_{\text {NSG }}$ represent the same entities as in SHA. From Fig. 2 we see that 0 to $n$ states represent the arrival rate for both SG and NSG users but $n$ to $s$ states are reserved only for the SG user. So if $\mathrm{SG}_{C h}$ represents the SG user channels and $s$ states represent total number of channels, then the remaining number of channels, for the NSG users, is given by:

$$
\mathrm{NSG}_{C h}=s-\left(\mathrm{SG}_{C h}\right)
$$

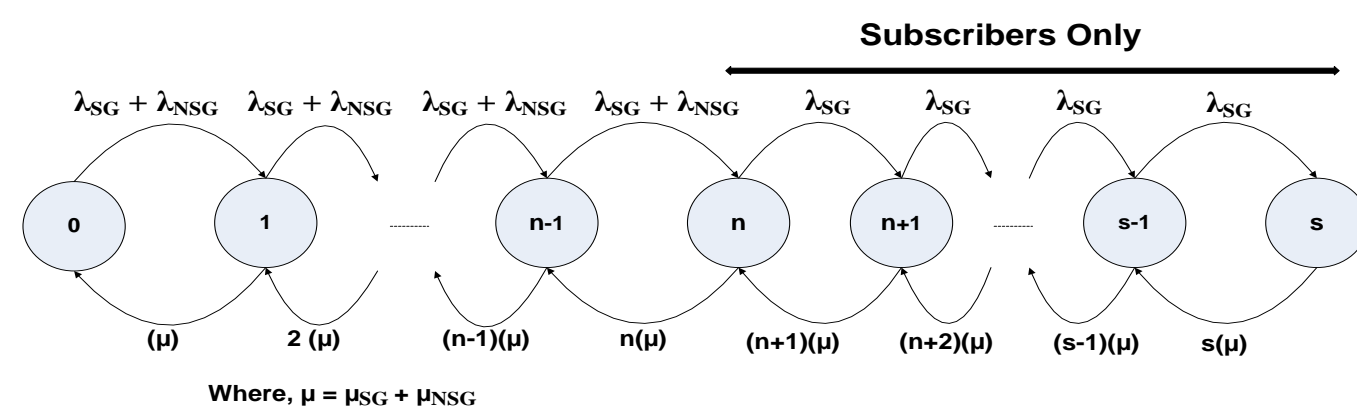

Fig. 2. Markov chain model for restricted hybrid access (RHA).

To find the total state probability of the $s$ states from Fig. 2 let us consider that $P_{j}$ is the statistical probability for total $j$ states, then for $0 \leq j<n$ the transition rate for $P_{j}$ to $P_{j+1}$ states is given by $\lambda_{\mathrm{SG}}+\lambda_{\mathrm{NSG}}$ and for $P_{j+1}$ to $P_{j}$ is given by $(j+1)\left(\mu_{\mathrm{SG}}+\mu_{\mathrm{NSG}}\right)$. From $n \leq j<s$ states, the transition rate for $P_{j}$ to $P_{j+1}$ is given by $\lambda_{\mathrm{SG}}$ because only the SG users can access these channels. The transition rate from 
$P_{j+1}$ to $P_{j}$ for $n \leq j<s$ is given by $(j+1)\left(\mu_{\mathrm{SG}}+\mu_{\mathrm{NSG}}\right)$. We calculate the blocking probabilities for the SG and NSG users as follows:

$$
\begin{gathered}
P_{j}=\left[\left(\frac{\lambda_{\mathrm{SG}}+\lambda_{\mathrm{NSG}}}{\mu_{\mathrm{SG}}+\mu_{\mathrm{NSG}}}\right)^{j} / j !\right] P_{o} \quad \text { for } 0<j \leq n \\
P_{j}=\left[\left(\left(\lambda_{\mathrm{SG}}\right)^{j-n}\left(\lambda_{\mathrm{SG}}+\lambda_{\mathrm{NSG}}\right)^{n}\right) /\left(j !\left(\mu_{\mathrm{SG}}+\mu_{\mathrm{NSG}}\right)^{j}\right)\right] P_{o} \text { for } n<j \leq s
\end{gathered}
$$

The total probability of the $s$ states is provided by eq. (7) and eq. (8). Like the SHA model, we need to find the probability of the zero state $P_{0}$ in order to calculate $P_{j}$. To find $P_{0}$ we solve eq. (2), eq. (7) and eq. (8) resulting in:

$$
P_{o}=1 /\left[\sum_{j=0}^{n}\left[\left(\frac{\lambda_{\mathrm{SG}}+\lambda_{\mathrm{NSG}}}{\mu_{\mathrm{SG}}+\mu_{\mathrm{NSG}}}\right)^{j} / j !\right]+\sum_{j=n+1}^{S} \frac{\left(\lambda_{\mathrm{SG}}\right)^{j-n}\left(\lambda_{\mathrm{SG}}+\lambda_{\mathrm{NSG}}\right)^{n}}{j !\left(\mu_{\mathrm{SG}}+\mu_{\mathrm{NSG}}\right)^{j}}\right]
$$

The blocking probability of the NSG users $P_{\mathrm{NSG}, b}$ is dependent on number of unavailable channels, so:

$$
P_{\mathrm{NSG}, b}=\sum_{j=n}^{S} P_{j}
$$

SG user blocking probability will be given by eq. (7)-eq. (9) and replacing $j$ by $s$ :

$$
\left.P_{\mathrm{SG}, b}=P_{s}=\left\{\left[\left(\frac{\lambda_{\mathrm{SG}}+\lambda_{\mathrm{NSG}}}{\mu_{\mathrm{SG}}+\mu_{\mathrm{NSG}}}\right)^{s} / s !\right]+\left[\left[\left(\lambda_{\mathrm{SG}}\right)^{s-n}\left(\lambda_{\mathrm{SG}}+\lambda_{\mathrm{NSG}}\right)^{n}\right] /\left[s !\left(\mu_{\mathrm{SG}}+\mu_{\mathrm{NSG}}\right)^{s}\right)\right]\right]\right\} P_{o}
$$

The arrival rates, $\lambda_{\mathrm{SG}}$ and $\lambda_{\mathrm{NSG}}$, are calculated by:

$$
\lambda=\rho \times \mu
$$

where, $\rho$ is the offered traffic and is equal to the total number of channels. In order to calculate the blocking probabilities; $P_{\mathrm{NSG}, b}$ and $P_{\mathrm{SG}, b}$ following algorithm is used:

Step A: Input:

1. Total number of channels (s states).

2. Percentage of the NSG Channels.

3. Percentage of the SG Channels.

Step B: Calculate $\lambda_{\mathrm{SG}}$ and $\lambda_{\mathrm{NSG}}$ using eq. (12) and eq. (13).

Step C: Calculate $P_{\mathrm{SG}, b}$ and $P_{\mathrm{NSG}, b}$ according to the proposed models in the subsections 2.1 and 2.2 .

\section{Simulation Results and Discussion}

\subsection{Simulation Results}

To simulate the models a single FAP is considered with a total range of 30 to 50 frequency channels. The service times, for which SG user and NSG user remains connected to the system, are given by $1 / \mu_{\mathrm{SG}}$ and $1 / \mu_{\mathrm{NSG}}$ respectively. The arrival rates $\lambda_{\mathrm{SG}}$ and $\lambda_{\mathrm{NSG}}$ are calculated by using eq. (12) according to the service arrival rates, i.e., $\mu_{\mathrm{SG}}$ and $\mu_{\mathrm{NSG}}=0.33 / \mathrm{min}$.

Fig. 3 shows the blocking probability curves for SG user channels by using the SHA model. The blocking probability curves are derived by varying the number of occupied channels by the NSG users. Three 
scenarios are created, i.e., $80 \%$ channels occupied by the NSG users, $40 \%$ channels occupied by the NSG users and 5\% channels occupied by the NSG users. By comparing these three scenarios it is shown that lower the percentage of occupied channel resources by the NSG users, lower will be the blocking probability of the SG users.

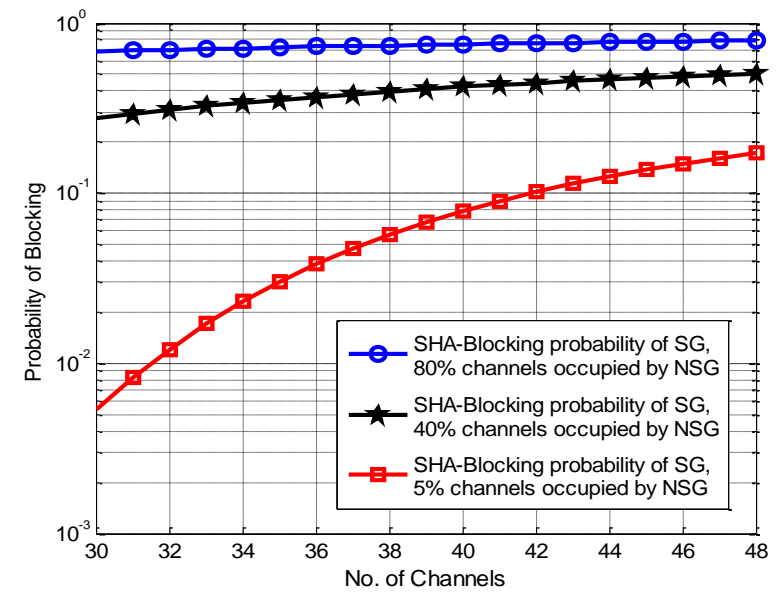

Fig. 3. Blocking probability curves for Subscriber Group, using SHA, against the total number of channels.

The total number of channels is set to 50 . Mean service times, $1 / \mu_{\mathrm{SG}}$ and $1 / \mu_{\mathrm{NSG}}$ are set to $3 \mathrm{~min}$.

Fig. 4(a) and Fig. 4(b) show the blocking probability curves for the both SG and NSG user channels by using the RHA model. It can be observed that, by reserving separate channels for the SG users and restricting the NSG users to gain access to the reserved channels, the blocking probability of the SG user channels is reduced to a negligible level. The curves shown in Fig. 4(a) and Fig. 4(b) are produced by creating scenarios of $20 \%$ reserved channels and $40 \%$ reserved channels for the SG users respectively. Further it is shown that by varying the number of reserved SG user channels, from $20 \%$ to $40 \%$, reduces the blocking probability of the SG user channels and increases the blocking probability of NSG user channels. The tradeoff here is only that if there are less number of SG users in FAP area then the reserved channel resources will be wasted, but the reduction in the blocking probability of SG channels is a major plus point and cannot be neglected.

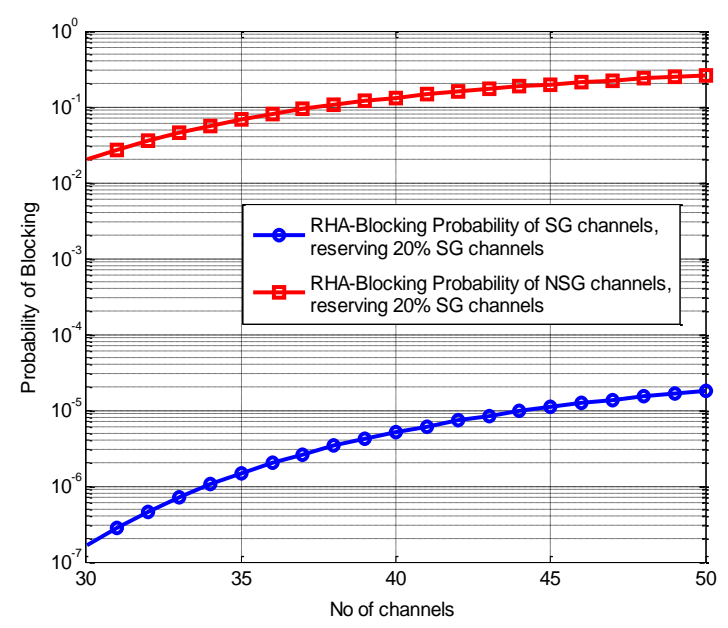

(a)

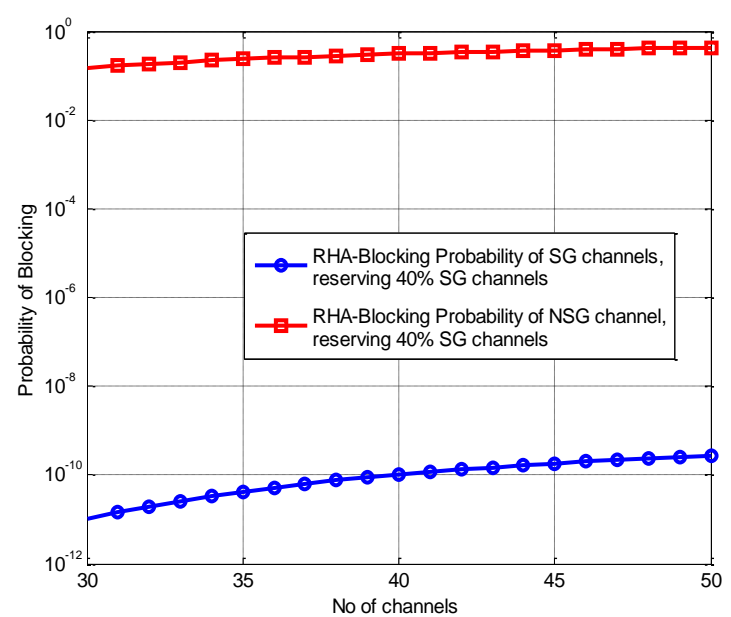

(b)

Fig. 4. Blocking probability curves for RHA against total number of channels. The total number of channels is set to 50 . Mean service times, $1 / \mu_{\mathrm{SG}}$ and $1 / \mu_{\mathrm{NSG}}$ are set to $3 \mathrm{~min}$. 
Here a question arises that for the RHA model why the blocking probability of the SG users is so low that it is almost negligible and that of the NSG users is too high as compared to the SG users? The answer is in two parts; the SG users blocking is so low because they have reserved channels which cannot be used by the NSG users and secondly if all the channels in the reserved part are busy they can occupy the channels from the non reserved portion, so the total probability becomes almost negligible.

\subsection{Discussion}

The idea of shared and restricted hybrid access, theoretically discussed in [5], has been extended and verified in this paper mathematically using Markov chain analysis and through simulations. Some important points of discussion from the QoS point of view are provided here:

SHA Model: From the simulations, we observe that if we have dense population of NSG users in the area, it can degrade the QoS of the SG users. SG users are of high priority because they pay extra for using additional resources e.g. high data rates or unlimited data usage etc. So, to deal with this kind of situation, SHA model will be best suitable for the low NSG user areas, so that NSG users occupy less channel resources and most of them are available to the SG users.

RHA Model: From the simulations, we see that by reserving the channels for SG users, their blocking probability can be controlled. This kind of model can perform better in dense populated NSG user areas, because they will not be able to occupy the channel resources of the SG users. This will improve the QoS of the SG users. One important matter is the efficient population estimation of the SG users. If the number of reserved channel resources for SG users are higher than the actual SG users present in the area, then most of the reserved channel resources will be wasted. As a result, the blocking probability of the NSG users will also increase in addition to the wastage of channel resources. So for better QoS, the analysis of the NSG and SG users in a certain coverage area must be done efficiently in a way that less channel resources are wasted.

\section{Conclusion}

In this work two models, SHA and RHA, have been proposed for the femtocell implementation in the next generation wireless networks. The NSG user and SG user blocking probabilities have been derived for both SHA and RHA. From the QoS point of view both models have their own significance. The analysis of SHA model shows that the coexistence of SG and NSG users with shared resources is possible but only under the circumstances of low NSG users else the QoS of the SG users will be degraded. It is shown through simulations that, in SHA, by increasing the NSG users the blocking probability of the SG users increases. On the contrary using the RHA models it is shown through the simulations that by reserving the channels resources the blocking probability of the SG users can be controlled. Further it is shown that by increasing the number of reserved channels, the blocking probability of SG users decreases significantly, which will

improve the QoS of the SG users. In this work only one FAP is considered for the blocking probability analysis but the proposed models can be tested using the real time data under different scenarios, e.g., using multiple FAPs, handover analysis and throughput analysis etc.

\section{Acknowledgment}

We thank MSIP (Ministry of Science, ICT and Future Planning), Korea, under the "Creative ICT Convergence Human Resource Development Program" support program and "Global IT Talent" support program (NIPA-2014-H0904-14-1005) supervised by the NIPA (National IT Industry Promotion Agency) for providing their support for this work.

\section{References}

[1] El-Sawy, H., \& Hossain, E. (December 2013). Channel assignment and opportunistic spectrum access in 
two-tier cellular networks with cognitive small cells. Proceedings of IEEE Globe Communication Workshops (pp. 4477-4482).

[2] Kim, J. S., \& Lee, T. J. (February 2010). Handover in UMTS networks with hybrid access femtocells. Proceedings of the 12th International Conference on Advanced Communication Technology: Vol. 1 (pp. 904-908).

[3] Chen, Y., Zhang, J., \& Zhang, Q. (May 2012). Utility-aware refunding framework for hybrid access femtocell network. IEEE Transactions on Wireless Communications, 11, 1688-1697.

[4] Lee, S. Q., Han, R. B. E., \& Park, R. B. (October 2011). Call admission control for hybrid access mode femtocell system. Proceedings of IEEE 7th International Conference on Wireless and Mobile Computing, Networking and Communications (pp. 512-516).

[5] Roche, G. de la, Valcarce, A., López-Pérez, D., \& Zhang, J. (2010). Access control mechanisms for femtocells. IEEE Communications Magazine, 48, 33-39.

[6] Jo, H.-S., Xia, P., \& Andrews, J. G. (2012). Open, closed, and shared access femtocells in the downlink. EURASIP Journal on Wireless Communications and Networking, 1-16.

[7] Radaydeh, R. M., \& Alouini, M. S. (June 2012). Switched-based interference reduction scheme for open-access overlaid cellular networks. IEEE Transactions on Wireless Communications, 11(6), 2160-2172.

[8] Wang, S. W., Huang, F. J., Yuan, M. D., \& Du, S. D. (February 2012). Resource allocation for multiuser cognitive OFDM networks with proportional rate constraints. International Journal of Communication Systems, 25, 254-269.

[9] Nguyen, K. D., Nguyen, H. N., \& Morino, H. (March 2013). Performance study of channel allocation schemes for beyond 4G cognitive femtocell-cellular mobile networks. Proceedings of IEEE Eleventh International Symposium on Autonomous Decentralized Systems (pp. 1-6).

[10] Choi, D., Monajemi, P., Kang, S., \& Villasenor, J. (November 2008). Dealing with loud neighbors: The benefits and tradeoffs of adaptive femtocell access. Proceedings of IEEE Global Telecommunications Conference (pp. 1-5).

[11] Valcarce, A., López-Pérez, D., Roche, G. de la, \& Zhang, J. (September 2009). Limited access to OFDMA femtocells. Proceedings of IEEE 20th International Symposium on Personal, Indoor and Mobile Radio Communications (pp. 1-5).

[12] Usman, M. R. (2009). Performance analysis of channel allocation schemes in Wimax. University Essay from Blekinge Tekniska Högskola.

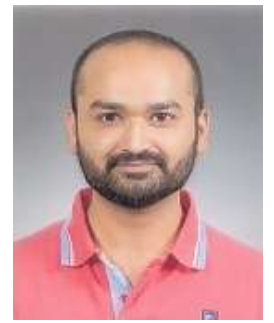

Muhammad Rehan Usman was born in Lahore City, Pakistan in 1986. He received the B.S. degree in electrical engineering from COMSATS Institute of Information Technology, Lahore, Pakistan, in 2008 and holds two M.S. degrees, one is the M.S. degree of electrical engineering from Blekinge Tekniska Hogkola, Sweden, in 2010 and another is the M.S. degree of project management from Kungliga Tekniska Hogskola (KTH), Sweden, in 2013. His research interests include MIMO, OFDM, NOMA, small cells and next generation networks $(4 \mathrm{G} / 5 \mathrm{G})$.

He was a lecturer in the Electrical Engineering Department at University of South Asia Pakistan from July 2012 to January 2013. Then he joined the Electrical Engineering Department of Superior University Lahore, Pakistan as a lecturer, from January 2013 to March 2014. He is now a Ph.D. research scholar at WENS Lab in School of Electronics in Kumoh National Institute of Technology since March 2014. 


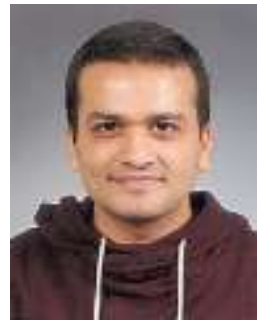

Muhammad Arslan Usman was born in Okara City, Pakistan in 1988. He received his B.S. degree in electrical engineering from COMSATS Institute of Information Technology, Lahore, Pakistan, in 2010 and received his M.S. degree in electrical engineering from Blekinge Tekniska Hogkola (BTH), Karlskrona, Sweden, in 2013. His research interests include MIMO, OFDM, NOMA, small cells, audio/visual signal processing and next generation networks $(4 \mathrm{G} / 5 \mathrm{G})$.

He has worked in Alcatel-Lucent Nigeria as a wireless technical pre-sales engineer (4G-LTE) from 2013-2014. After serving in Alcatel-Lucent for a year he is now a PhD research scholar at WENS Lab in School of Electronics in Kumoh National Institute of Technology since September 2014.

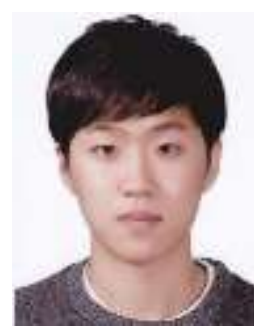

Sang Hoon Lee was born in Gumi City, The Republic of Korea, in 1988. He received his B.S. degree in electrical engineering from Kumoh National Institute of Technology, Gumi, The Republic of Korea, in 2013. His research interests include coexistence among networks, MIMO, OFDM, NOMA, small cells and next generation networks (4G/5G).

He is working as a master's student at WENS Lab in School of Electronics in Kumoh National Institute of Technology since March 2013.

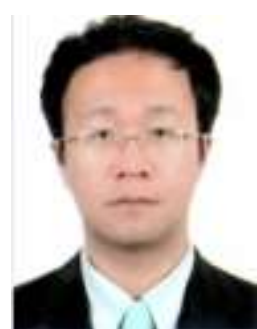

Soo Young Shin was born in 1975. He received his B.S., M.S., and Ph.D. degrees in electrical engineering and computer science from Seoul National University, Korea in 1999, 2001, and 2006, respectively. His research interests include wireless LAN, WPAN, WBAN, coexistence among wireless networks, industrial and military network, cognitive radio, MIMO, OFDM, NOMA and next generation mobile wireless broadband networks $(4 \mathrm{G} / 5 \mathrm{G})$.

He was a visiting scholar in FUNLab at University of Washington, US, from July 2006 to June 2007. After 3 years working in WiMAX Design Lab of Samsung Electronics, he has been an assistant professor in the School of Electronics in Kumoh National Institute of Technology since September 2010. 